\title{
Hospitals as a 'risk environment: An ethno-epidemiological study of voluntary and involuntary discharge from hospital against medical advice among people who inject drugs
}

\author{
Ryan McNeil ${ }^{1}$, Will Small ${ }^{1,2}$, Evan Wood ${ }^{1,3}$, and Thomas Kerr ${ }^{1,3}$ \\ ${ }^{1}$ British Columbia Centre for Excellence in HIV/AIDS, St. Paul's Hospital, 608-1081 Burrard \\ Street, Vancouver, BC, Canada, V6Z 1 Y6 \\ ${ }^{2}$ Faculty of Health Sciences, Simon Fraser University, Burnaby, BC, Canada \\ ${ }^{3}$ Department of Medicine, University of British Columbia, Vancouver, BC, Canada
}

\section{Abstract}

\begin{abstract}
People who inject drugs (PWID) experience high levels of HIV/AIDS and hepatitis C (HCV) infection that, together with injection-related complications such as non-fatal overdose and injection-related infections, lead to frequent hospitalizations. However, injection drug-using populations are among those most likely to be discharged from hospital against medical advice, which significantly increases their likelihood of hospital readmission, longer overall hospital stays, and death. In spite of this, little research has been undertaken examining how social-structural forces operating within hospital settings shape the experiences of PWID in receiving care in hospitals and contribute to discharges against medical advice. This ethno-epidemiological study was undertaken in Vancouver, Canada to explore how the social-structural dynamics within hospitals function to produce discharges against medical advice among PWID. In-depth interviews were conducted with thirty PWID recruited from among participants in ongoing observational cohort studies of people who inject drugs who reported that they had been discharged from hospital against medical advice within the previous two years. Data were analyzed thematically, and by drawing on the 'Risk Environment' framework and concepts of social violence. Our findings illustrate how intersecting social and structural factors led to inadequate pain and withdrawal management, which led to continued drug use in hospital settings. In turn, diverse forms of social control operating to regulate and prevent drug use in hospital settings amplified drug-related risks and increased the likelihood of discharge against medical advice. Given the significant morbidity and health care costs associated with discharge against medical advice among drug-using populations, there is an urgent need to reshape the social-structural contexts of hospital care for PWID by shifting emphasis toward evidence-based pain and drug treatment augmented by harm reduction supports, including supervised drug consumption services.
\end{abstract}

\footnotetext{
(c) 2014 Elsevier Ltd. All rights reserved

Corresponding Author: Thomas Kerr, PhD British Columbia Centre for Excellence in HIV/AIDS 608- 1081 Burrard Street Vancouver, BC V6Z 1Y6 uhri-tk@cfenet.ubc.ca.

Publisher's Disclaimer: This is a PDF file of an unedited manuscript that has been accepted for publication. As a service to our customers we are providing this early version of the manuscript. The manuscript will undergo copyediting, typesetting, and review of the resulting proof before it is published in its final citable form. Please note that during the production process errors may be discovered which could affect the content, and all legal disclaimers that apply to the journal pertain.
} 


\section{INTRODUCTION}

Current estimates suggest that more than 15 million people worldwide regularly inject drugs (Mathers et al., 2008). The health sequelae of injection drug use can be severe, and include infectious disease acquisition and other direct complications of injecting (e.g., overdose). As a consequence, people who inject drugs (PWID) suffer from disproportionately high levels of HIV/AIDS (Mathers et al., 2008) and hepatitis C (HCV) infection (Aceijas \& Rhodes, 2007) that, together with high rates of non-fatal overdose (Warner-Smith et al., 2002), injection-related soft tissue infections (Cooper et al., 2007; Lloyd-Smith et al., 2008), and other co-morbidities common among this population, lead to frequent hospitalizations (Gebo et al., 2003; Kerr et al., 2005; Palepu et al., 2001). As a result, PWID are admitted to hospital significantly more often than the general age-adjusted population (Kerr et al., 2005).

There is also clear evidence that PWID are one of the populations most likely to be discharged from hospital against medical advice (Anis et al., 2002; Choi et al., 2011; Jeremiah et al., 1995; Yong et al., 2013). For our purposes, discharges against medical advice are understood to be inclusive of discharges occurring among patients who have left hospital prior to completing treatment (whether they have notified hospital staff they are leaving or not), as well as those who have been involuntarily discharged prior to completing treatment (e.g., discharge for breach of hospital policies). Discharges from hospital against medical advice among PWID can exacerbate health complications, and this population is significantly more likely to be readmitted for the same condition and have longer eventual hospital stays than those who have completed treatment (Anis et al., 2002; Choi et al., 2011; Glasgow et al., 2010; Hwang et al., 2003). Furthermore, those discharged against medical advice are at an increased risk of mortality (Choi et al., 2011; Yong et al., 2013), with one Canadian study finding that this population is approximately three times as likely to die in the year following their initial discharge (Choi et al., 2011).

Whereas epidemiological analyses of hospital admissions and discharge data have identified crude demographic risk factors for departures against medical advice among PWID, including female gender, younger age, and Aboriginal ancestry (Anis et al., 2002; Chan et al., 2004), comparatively less attention has been paid to contextual forces underlying this phenomenon. Several studies have noted that these departures are most likely to occur in the days immediately surrounding the disbursement of social assistance payments (Anis et al., 2002; Riddell \& Riddell, 2006), and that these may be mitigated to some degree by providing access to inpatient methadone maintenance treatment (Chan et al., 2004). However, the lack of attention to the potential role of intersecting social, structural, and environmental forces operating within hospitals in shaping discharges against medical advice among PWID means that these explanations are incomplete. In addition, these individual-level explanations primarily attribute discharges against medical advice to 'active drug use' in a manner that risks locating responsibility for these outcomes solely with PWID. This overlooks social and structural-environmental characteristics of hospitals that potentially lead to discharges against medical advice, and creates a missed opportunity to modify these environmental characteristics to promote better retention of PWID in care.

This research gap is particularly striking given more than a decade of evidence demonstrating the need for increased attention to 'risk environments' - that is, social and physical settings in which factors exogenous to the individual (i.e., social situations, structures, and places) interact to produce or reduce drug-related harms (Rhodes et al., 2005; Rhodes, 2009). The emergence of the 'risk environment framework' has focused attention on how the interplay between physical, social, economic, and policy factors operating across the micro-, meso-, and macro-environmental levels produce harm among PWID (Rhodes, 2002; Rhodes, 2009). Concepts of structural vulnerability and everyday violence have 
further proven instructive in framing the suffering experienced by drug-using populations (Fairbairn et al., 2008; Shannon et al., 2008). Structural vulnerability refers to how social arrangements embedded in the organization of society render particular populations disproportionately vulnerable to harm (Quesada et al., 2011). Everyday violence refers to the normalization of suffering within any particular context due to the contextual forces that render it invisible (Scheper-Hughes, 1992). Together, these concepts give focus to how the structural context of drug use (e.g., drug criminalization) produces vulnerability to an array of drug- and health-related harms, and reflect dominant power structures that normalize these harms as the natural consequences of drug use. Sorting out the complex contextual forces operating within the risk environments of people who use drugs is critical to understanding their role in shaping health outcomes - in this case, discharges against medical advice - and informing social-ecological interventions.

While qualitative research into the experiences of injection drug-using populations in hospital settings is limited, and has yet to systematically explore the experiences of those discharged against medical advice, it has generated preliminary insights into the social forces operating within the hospital 'risk environment' (Berg et al., 2009; Merrill et al., 2002; Neale et al., 2008). In an ethnographic study exploring patient-physician interactions in an American urban teaching hospital, Merrill and colleagues (2002) outlined how `mutual mistrust' frames the hospital care of PWID. Whereas physicians attributed their difficulty in managing pain to the fear of being 'deceived' by 'drug-seeking' patients and the lack of clinical protocols for pain management among injection drug-using populations, PWID viewed physicians with suspicion and believed that their treatment was primarily shaped by discrimination (Merrill et al., 2002). Whether or not this, together with other contextual factors, plays a role in discharges from hospital against medical advice warrants further attention.

These issues are of considerable relevance in Vancouver, Canada, the site of a longstanding injection drug use epidemic and home to an estimated 15,000 PWID (McInnes et al., 2009).

The majority of the city's injection drug-using population will visit an emergency department each year (Kerr et al., 2005), with approximately $17 \%$ of these visits resulting in hospitalization (Fairbairn et al., 2011; Palepu et al., 2001). In Vancouver, PWID are covered by universal, publicly-funded health care insurance. However, while comprehensive harm reduction services, including a supervised injection facility, are integrated into the local public health system and local hospitals struggle with the optimal management of hospitalized PWID, hospitals generally operate under abstinence-based drug use policies (Providence Health Care, N.D; Vancouver Coastal Health, 2008). While abstinence-based policies in part reflect anti-drug laws, they are also framed as necessary in promoting patient and staff safety. In addition, prescribing principles promoted by the British Columbia College of Physicians and Surgeons regarding prescription opioids have been primarily developed for non-drug-using populations, and warn against prescribing opioids to 'highrisk' populations (British Columbia College of Physicians and Surgeons, 2012). Past research has shown that PWID in this setting are frequently discharged from hospital against medical advice (Anis et al., 2002; Choi et al., 2011; Palepu et al., 2001), and in one urban teaching hospital account for more than half of such discharges (Choi et al., 2011).

We undertook this ethno-epidemiological study to explore how intersecting social, structural, and environmental forces shape the experiences of PWID in hospitals settings and contribute to discharges against medical advice. We were particularly concerned with the role of abstinence-based drug policies in hospital settings in framing the social and structural-environmental contexts of hospital care, pain management practices, and inhospital drug use. Finally, we aimed to identify ways in which the hospital `risk 
environment' could be modified to minimize the potential for adverse outcomes, including discharges against medical advice.

\section{METHODS}

This ethno-epidemiological study was undertaken in connection with two ongoing prospective cohort studies: the Vancouver Injection Drug Users Study (VIDUS) and the AIDS Care Cohort to Evaluate Exposure to Survival Services (ACCESS). These cohort studies include more than 2000 current and former drug users, and their methods have been described in detail elsewhere (Strathdee et al., 1997; Wood et al., 2003). Ethnoepidemiology seeks to uncover how social meanings and contexts influence patterns of drug and health harms by merging epidemiological and qualitative methods (Lopez et al., 2013; Wagner et al., 2012). Increased understanding of the contextual forces that shape patterns and distributions of harm is critical to informing the development of structural and environmental interventions to minimize harms (Rhodes et al., 2006). Between December 2011 and February 2013, we undertook qualitative interviews with thirty cohort participants who reported that they had recently been discharged from hospital against medical advice during follow-up surveys that are part of their participation in the aforementioned cohort studies. All study activities were approved by the Providence Healthcare/University of British Columbia Research Ethics Board.

\section{Participant recruitment}

Cohort participants were eligible for participating in this study if, during routine follow-up surveys completed in the past two years, they answered "yes" to the following question: "In the past six months, did you leave hospital before your treatment was complete?" We chose this follow-up period to ensure an adequate pool of potential participants, while minimizing biases due to poor recall of events. We used recruitment quotas to ensure that women and people of Aboriginal ancestry were adequately represented in our sample. Eligible participants were identified through database queries of cohort data and contacted by study personnel, who described this study and invited them to participate in an interview. Study personnel also recruited those who reported that they had been discharged against medical advice during follow-up surveys administered over the course of our study.

\section{Data Collection}

Interviews were conducted by the lead author (RM) at the cohort study office. Prior to the interview, the lead author explained the study to participants, answered questions, and obtained written informed consent. There were no refusals to participate and no dropouts. Participants each received a $\$ 20$ CAD honorarium upon completion of the interview. Interviews were facilitated through the use of an interview topic guide adapted from previous qualitative work on health care access among PWID (Krusi et al., 2009; Small et al., 2008), and revised to include questions specific to our study objectives. This interview topic guide aimed to facilitate discussion regarding how contextual forces shape experiences in hospitals and lead to discharges against medical advice. Interviews were audio recorded and averaged approximately 45 minutes in length. Interviews were transcribed verbatim and reviewed for accuracy by the lead author.

\section{Data Analysis}

Data collection and analysis took place concurrently, and emerging themes informed lines of inquiry during subsequent interviews. We imported interview transcripts into NVivo (version 9) to facilitate data management and coding. Transcripts were coded thematically using an inductive and iterative process (Corbin \& Strauss, 2008), and regular meetings were held to discuss emerging themes. Once the final themes were established, the lead 
author re-coded sections of the transcripts to enhance the reliability and validity of these categories. To advance beyond thematic description, we then drew upon the risk environment framework and concepts of structural vulnerability and everyday violence to interpret our themes.

\section{Sample Characteristics}

Thirty individuals participated in in-depth interviews, including sixteen men, thirteen women, and one transwoman. Participants were an average of 45 years of age (range 29-59 years). Seventeen participants reported Aboriginal ancestry, while the remaining participants self-identified as Caucasian $(\mathrm{n}=12)$ and African Canadian $(\mathrm{n}=1)$. Half of our participants were living with HIV, while twenty-two had been diagnosed with HCV. Five participants living with HIV reported suboptimal adherence to highly-active antiretroviral therapy (HAART) prior to hospitalization, while another five reported that they were not taking HAART. Prior to their most recent hospitalization, participants reported that they lived in single room occupancy hotels $(n=17)$, non-market housing $(n=5)$, emergency shelters $(n=3)$, or were unhoused $(n=5)$. All participants had a history of injection drug use, and twenty-two currently injected drugs. All participants had used drugs in the thirty days prior to their most recent hospitalization, with crack cocaine $(n=22)$, heroin $(n=18)$, powdered cocaine $(n=12)$, and prescription opioids $(\mathrm{n}=7)$ identified as the most commonly used drugs. The most commonly reported reasons for hospitalization were injection-related infections $(n=8)$, pneumonia $(n=5)$, and traumatic injury $(n=4)$. Nearly all participants $(n=28)$ reported multiple hospitalizations within the past five years, with half reporting four or more hospitalizations.

\section{RESULTS}

\section{“They felt maybe I was getting high" - 'Drug-seeking' and pain management}

Our analysis of participant accounts underscored the role of social and structural forces in shaping pain management practices and producing suffering that framed experiences in hospital settings. All participants reported co-morbidities that, together with their presenting illness or injury, resulted in complex pain management needs. The vast majority of our participants $(n=25)$ had histories of opiate dependency, and those who were injecting heroin or prescription opioids at the time of their hospitalization indicated that their pain management needs were compounded by 'dopesickness' - that is, the extreme discomfort and pain accompanying opiate withdrawal. Participants described their level of pain as "excruciating" and "like being stuck with electric volts", with those experiencing dopesickness adding that they felt "nauseous" and like they were "coming unglued". The following excerpts highlight experiences of pain and opiate withdrawal, respectively, typical among our participants:

It was really, really bad at the beginning...I got shots of pain that were so severe actually cried... I had to cry out more once or twice within two or three hours. It was really, really excruciating. Every fifteen minutes or so, like, the pain was just unbelievable. [Participant \#29, African-Canadian Male, 53 years old]

I was constantly tired, no energy. Nauseous all the time, vomiting, couldn't eat, and [had] the runs. My eyes were leaking all the time. My nose was leaking. To me, that was all withdrawal symptoms. [Participant \#13, Caucasian Female, 44 years old]

With very few exceptions, however, participants reported that their pain management needs were unmet. Participant accounts underscored how narcotics control (macro-structural force) intersected with stereotypes of 'drug-seeking addicts' (macro-social force) to frame pain 
management practices in hospitals. Whereas these intersecting social-structural forces are situated within larger cultural discourses characterizing drug use as 'immoral', 'irresponsible', and 'pleasure-seeking', participants positioned their 'dopesickness' as a legitimate medical concern and emphasized how opiate maintenance was necessary to "get better" or "get straight". Furthermore, even among those experiencing opiate withdrawal, most participants viewed narcotics as necessary to alleviate the pain caused by their primary diagnoses (e.g., osteomyelitis, broken ribs).

Our findings underscore how the perception that participants were 'drug-seeking' was critical in shaping the social-environmental context of hospital care, and likely delegitimized the very real pain and suffering that they endured. Nearly all participants spoke of how they were routinely denied pain medication or given dosages that did not account for heightened tolerance. Some participants spoke of how physicians and nurses dismissed their concerns and characterized them, in the words of one participant, as "just another junkie addict looking for free drugs". Many participants of Aboriginal ancestry further expressed that institutionalized racism reinforce the view among hospital staff that they were `drugseeking'. This approach to pain management was experienced as a form of everyday violence, in that the pain and suffering experienced by participants was normalized as a natural part of their care that was reproduced through the routine denial of pain medication. For example:

I was in pain and I didn't want to bitch about being in pain||The last time I went in there and I told them, "Excuse me, I'm very sore and it's taking forever. Could I please see somebody or get something?" And, they said, "We can't give you anything until you see a doctor." [...] Because I was there often with this problem [abscess in leg], they thought I was looking for pain pills. [Participant \#11, Aboriginal Female, 51 years old].

They [nurses] were very combative with me. When I was done my surgery, it was very painful and I needed my morphine upped because it just wasn't working...I can only guess and say that maybe they felt maybe I was getting high or something. I kept trying to tell them that it's not enough...I had major surgery on my colon. I couldn't even move...The nurses wouldn't listen to me they're. I felt they didn't believe me and they were a little combative in speaking with me. [Participant \#20, Aboriginal Male, 45 years old]

Furthermore, the minority of participants $(n=6)$ who were administered methadone in hospital reported disruptions in their regular dosing schedules and amounts. While these participants acknowledged that hospital staff lacked experience in methadone maintenance treatment, they also expressed that methadone withdrawal was a low priority among nurses and physicians.

I have to have methadone every day. I'm on fifty milligrams and, if I don't have it, I'm in bad shape. I kept asking them, "I take it at five thirty every morning and I want it." [...] Well, they did it whenever they felt like it. They don't understand that methadone is keeping me functional. [...] I wanted to get the heck out of there.

[Participant \#24, Caucasian Female, 55 years old]

\section{"Between a rock and a hard spot" - The need to manage pain and withdrawal}

Most participants expressed that heroin and prescription opioid injection were the only avenues available to them to address pain and withdrawal. While several participants attributed their continued drug use to the craving associated with cocaine use, the vast majority indicated that drug use during their hospitalization was primarily motivated by the need to manage pain and opiate withdrawal. Whereas some participants expressed that it 
was not "normal" to inject heroin or prescription opioids while hospitalized, in that it contradicted the 'curative' nature of hospital care, they nonetheless emphasized the anticipated relief from pain and opiate withdrawal. In some cases, participants articulated how pain and withdrawal symptoms directly interfered with treatment, and that only after addressing these immediate needs would they be 'strong' enough to recover. As one participant explained:

I had to get out of there while I could move because I was losing so much weight... When I begged and begged to get some help [i.e., prescription opioids], they couldn't, weren't gonna do anything and so I just said, "Fine, I'm leaving." [...] I was concerned [about the health consequences of leaving hospital]. You know, I got this other thing [opiate dependency] and it's...it's like you're stuck between a rock and a hard spot. I mean, how can I even fight off the infection if I can't stop puking and shitting? [Participant \#15, Caucasian Female, 47 years old]

At its most extreme, one participant articulated how his transition to heroin injection was the consequence of inadequate pain management in hospital settings, and represented this as the only means to address the extreme pain associated with osteomyelitis (i.e., an infection of the bone or bone marrow).

I started using heroin when I had that osteomyelitis. I was in so much pain and morphine wasn't cutting it... [The reason why] I started using the heroin actually was to kill the pain. [...] The pain and the osteomyelitis...it was so crippling I felt, like, so stiff... If they [hospital staff] had given me the right dose, I probably wouldn't even be using heroin... [The morphine] wasn't enough and they weren't giving it into my I.V. They were shooting it into my muscle and that wasn't killing my pain... The heroin I was using, I'd do right into my bloodstream and it would kill the pain. [Participant \#5, Aboriginal Male, 44 years old]

\section{"Like I was in jail" - Surveillance, regulation, and in-hospital drug use}

Whereas the social-structural context of pain management practices in hospitals perpetuated the need to inject drugs, participant accounts underscored how larger structuralenvironmental context of hospital settings was shaped by the enforcement of abstinenceonly drug policies (macro-structural environmental factor). Participants characterized hospitals as "jails" or "prisons", and viewed hospital staff as playing the role of "cop" in enforcing abstinence-only drug policies. Nearly all participants described how some nurses and security guards subjected them to surveillance and regulation by "policing" their drug use. Many participants reported that some nurses closely monitored their behavior for outward signs that they were injecting drugs in hospital. Other participants reported that they were subjected to physical searches by security guards when suspected of possessing drugs. As one participant explained:

[Security guards] yell and scream at you... When there's nobody around, [they say], "You fucking junkie." [...] A few times, I've been shaken down [searched] by [security guards] even though [I had] nothing to get high [i.e., had no drugs in her possession]. They search you, destroy your property, cause a scene, and make sure everybody there knows that you're a drug addict. [...]They use their authority to pull power trips more or less. It's not right. [Participant \#12, Aboriginal Female, 29 years old $]$

These forms of surveillance reinforced participants' marginal status as 'drug addicts'. Participants expressed dissatisfaction with these forms of surveillance and regulation, with one participant describing that they made her feel "like I was in jail." 
Rather than preventing drug use, this surveillance and regulation produced structural vulnerability to drug and health harms. Most participants continued to use drugs during the period of their hospitalization to cope with pain and opiate withdrawal or due to craving associated with cocaine use, and half of our participants reported in-hospital drug use. The strategies enacted to avoid detection by nurses and security guards compromised participants' ability to practice harm reduction. Many participants expressed that they could not request syringes without attracting suspicion or risking involuntary discharge. Some participants subsequently reused syringes that they had snuck into hospital and hidden in their personal belongings, and which were potentially contaminated with bacteria which could further cause osteomyelitis or cellulitis (i.e., a bacterial skin infection). As one participant with recurring injection-related infections explained, "I always try to have a rig on me... [The nurses] don't know I have them. [I keep it] in my coat, a bag or whatever I brought". Other participants relied on visitors to bring injecting equipment, and subsequently injected with syringes of an unknown origin. One participant explained the challenges and risks associated with accessing syringes in hospitals:

They [i.e. nurses] don't give rigs [i.e. syringes] to us...I think that they should. If not, we're reusing our rigs or we're having to risk getting kicked out for stealing them or people'll be sharing them. [...] I know one girl was using her same rig for days to the point where it was tearing and she was suffering every time she'd do her fix. She just didn't have it in her to go and try and steal clean rigs. Whereas for me, my friend that I was with had no problem. She would just sneak in and grab some for both of us. [Participant \#30, Aboriginal Female, 28 years old]

The most common strategy to avoid detection when injecting was to use drugs in locked hospital washrooms. Very few of our participants had been assigned private rooms, and instead shared rooms with up to five other patients. Participants indicated that washrooms were one of the only spaces over which they could exert control and thus evade surveillance. Despite widespread awareness among local PWID of the overdose risks associated with injecting alone, the situated risk perceptions of our participants elevated other concerns (e.g., pain management, avoiding detection) above the need to mitigate these risks. For example:

If you're sharing a room with somebody, there's always that threat that somebody's just gonna come in and not realize you're in there [the bathroom] and open [the door]. [...] I think they pretty much have zero tolerance in [the hospital]. I was worried about getting kicked out and then not getting the proper health care that I needed to get better. [...] I'd turn the tap so, if they came in my room to check to see if I was okay, then they'd hear the water running so they'd figure oh she's just in the bathroom. [Participant \#25, Caucasian Female, 44 years old]

\section{"I'Il just run out" - Well-intentioned departures from hospital}

Approximately half of our participants reported that they left hospital altogether when using drugs. Some participants "snuck out" of the hospital in the hope that they would return before nurses discovered they had gone. Other participants had been admitted to hospital wards that regularly issue "day passes", which allow patients to leave hospital for a specified amount of time (typically up to six hours) at the discretion of nurses or physicians without being discharged. Day passes were used by some wards to accommodate ongoing drug use, in that it was expected that participants would consume drugs off-site and not return to hospital until the immediate effects of intoxication had subsided. Most homeless or unstably housed participants stayed close to the hospital and used drugs in nearby public settings (e.g., parks, alleyways), while housed participants returned to their residence. For example:

I only had a pass so my plan was just to go grab some dope and then go back to the hospital. [I] stopped a couple of blocks away from there to do some dope. [I] just 
ended up staying in that one little spot in the park there and getting high for the day and then went and checked back in. [Participant \#9, Caucasian Male, 48 years old]

While nearly all participants intended to return to hospital, most did not return until after their passes had expired (resulting in discharge) and many did not return at all. Several participants emphasized how their health deteriorated after leaving hospital. For example:

The first time it was just, "OK, I'll just run out. They won't know I'm gone.” And then, I got stuck out there. Like, I was to a point where I wouldn't stop [binge cocaine use]. And then, I finally came back the next day 'cause I was afraid of losing my leg [due to a soft tissue infection]. [...] And then, the second time [I left hospital], I couldn't walk at all and my leg swelled up twice the size and there was pus draining out of it. [Participant \#13, Caucasian Female, 44 years old]

Some participants later returned to the same hospital to resume treatment and were readmitted through the emergency department. These participants reported that, subsequent to returning to hospital, they were subjected to greater scrutiny by some nurses, with several noting that they were denied day passes.

\section{'Get the fuck out' - Involuntary discharge for in-hospital drug use}

Whereas all participants indicated that they had been admitted to hospital for complex health problems, and required extensive treatment, approximately one third of our participants reported that they were involuntarily discharged for in-hospital drug use. Participant accounts underscored how, while many injected in locked bathrooms in an attempt to conceal their drug use, these were highly-regulated spaces that were actively monitored by nurses and security guards. In turn, most participants reported that they had been involuntarily discharged after they were caught, or suspected of, injecting drugs in bathrooms. For example:

I went to use the bathroom, and they sent the police in the bathroom. They said I was taking too long and they thought I was using drugs in there. I was on the toilet, and the cop walks in with the key. [...] He says, "You're taking too long. Get the fuck out." He's swearing at me. He's standing there with the security guards, and couple of the staff from the hospital. [...] They physically escorted me out. They told me they were going to arrest me if I step back on the property. They said I was creating a disturbance. [...] I was just using the bathroom. [Participant \#1,

Aboriginal Male, 39 years old]

Some participants acknowledged that they were disruptive during these encounters (e.g., swearing at hospital staff), which likely discouraged nurses and security guards from exercising discretion (i.e., seizing and disposing of the drugs but stopping short of involuntary discharge). However, these participants did not wish to discontinue treatment and expressed concern regarding the potential health consequences of involuntary discharge, and nearly all were later re-hospitalized.

\section{DISCUSSION}

In summary, our findings highlight how abstinence-only drug policies, together with inadequate pain management fuelled by narcotics control and negative stereotypes, frame hospital care, and produce structural vulnerability to harm among PWID. Diverse forms of social control that function to regulate drug use in hospitals (i.e., surveillance and regulation) increase the potential for drug-related harm and discharges against medical advice. Our findings demonstrate that hospitals constitute not just a setting to receive treatment and care for PWID, but a 'risk environment' where social and structural conditions 
produce discharges against medical advice and, in turn, more complicated and protracted medical treatment.

Conceptualizing hospitals as 'risk environments' allows us to better appreciate how contextual forces operating within hospitals shape diverse harms, including discharges against medical advice, and advance beyond individualized approaches that associate risk with moral culpability and lack of awareness of potential consequences (Rhodes, 2002). Consistent with research in drug scene milieus (Rhodes et al., 2007; Small et al., 2007), our findings demonstrate the role of the criminalization of drug use in perpetuating systems of social control that render PWID vulnerable to harm. Previous research on public injection settings has described how street policing, together with the stigma associated with injection drug use, leads to a sense of urgency when injecting that compromises PWID's ability to follow harm reduction practices (Rhodes et al., 2007; Small et al., 2007). Although hospitals are distinct from typical public injection settings in many ways, these same social and structural forces shape hospital care, and similarly constrain PWID's ability to practice harm reduction. Notably, we found that PWID went to extreme measures to conceal in-hospital drug use, and thereby minimize their likelihood of being caught and involuntarily discharged. Several of these measures (e.g., injecting alone in locked washrooms, injecting with syringes of an unknown origin) dramatically increase the risk of fatal overdose or HIV/ $\mathrm{HCV}$ transmission. In this regard, our findings highlight the importance of considering how diverse settings constitute risk environments for injection drug-using populations, and how drug criminalization frames the structural vulnerability of PWID in these settings.

Although it has been widely reported that complex co-morbidities, along with inadequate pain management, contribute to high levels of unmanaged pain among PWID (Neighbor et al., 2011; Passik et al., 2006), only limited attention has been paid to how untreated and undertreated pain shapes experiences in hospital settings (Merrill et al., 2002; Neale et al., 2008). Previous research has underscored the complexities of pain management among PWID, and emphasized how physician's perceptions of people who use drugs shape prescribing practices (Berg et al., 2009; Merrill et al., 2002). In a qualitative study of physician experiences treating chronic pain among PWID, Berg and colleagues (2009) found considerable variation in prescribing practices, and explored how this was influenced by larger discourses that characterize PWID as 'drug-seeking'. Many physicians consciously undertreated pain because they were concerned about promoting continued drug use (Berg et al., 2009).

Our findings build upon this research by demonstrating how this powerful cultural stereotype shapes hospital care for PWID and possibly leads to treatment decisions that increase the likelihood of discharges against medical advice. Furthermore, by documenting how the stereotype of the 'drug-seeking addict' intersected with institutionalized racism, our findings in part explain previous epidemiological findings indicating that Aboriginal ancestry increases the likelihood of discharge from hospital against medical advice among drug-using populations (Anis et al., 2002; Chan et al., 2004). Importantly, our participants described how inadequate pain management was normalized within hospital settings, and characterized their treatment by nurses and physicians as complicit in perpetuating suffering. This approach to pain management may be understood as a manifestation of the 'everyday violence' endured by PWID and underscores the urgent need to rethink pain management practices for drug-using populations and reorient them toward alleviating suffering. While further reforms may be needed to accommodate this, including changes to legal and professional regulations regarding the prescribing of prescription opioids, it is important to consider the high potential for negative outcomes if these changes are not made.

Furthermore, given the central role of discrimination in shaping pain management practices, 
education and training programs aimed at improving cultural competency among hospital staff are likely needed.

Greater acknowledgement that contextual forces operating within hospital settings produce suffering, and contribute to discharges against medical advice, necessarily begs the question of whether changes to the environmental contexts of hospital settings can improve care for drug-using populations. Our findings illustrate how injection drug-using populations undergo a vicious cycle of emergency department visits, hospitalizations, and departures that repeats itself to both increase the risk of death and the overall burden on the health care system. Certainly, there is an urgent need to integrate evidence-based approaches that show promise in disrupting this cycle. Methadone maintenance treatment has shown some promise in mitigating departures from hospital prior to completing treatment among PWID (Chan et al., 2004), and efforts to increase access to opiate substitution therapies and appropriate pain management among hospitalized PWID are needed. To achieve this goal, increased addictions and pain management training among physicians and nurses will be necessary (Miller et al., 2001).

Over the past decade, considerable evidence has mounted highlighting the role of `safer environment' interventions (e.g., syringe exchange programs, supervised drug consumption facilities) in reshaping the 'risk environment' of people who use drugs (Rhodes et al., 2006). Within this context, supervised drug consumption facilities have been found to be particularly effective in creating social, structural, and environmental conditions that enable harm reduction practices and facilitate access to health care services (Kerr et al., 2007; Krusi et al., 2009; Small et al., 2009; Small et al., 2008). Moreover, preliminary evidence suggests that this harm reduction strategy has significant potential to reshape the social and structuralenvironmental contexts within health care settings (Krusi et al., 2009). However, hospital policies, including those existing where the present research was conducted, continue to be primarily oriented toward promoting and, in some cases, enforcing drug abstinence. Against this backdrop, our findings lend support to the argument for integrating comprehensive harm reduction approaches, including supervised drug consumption services, into hospitals (Rachlis et al., 2009). While this approach is by no means a panacea, our findings suggest that this has the potential to reduce the deleterious effects of efforts to deter and limit drug use within hospital settings, and thus drug-related risks (e.g., injecting alone) and discharges from hospital against medical advice. This approach would allow hospital staff to shift their attention from policing drug use to more pressing patient concerns, and also minimize the conflicts occurring in hospitals.

We acknowledge that this approach may encounter opposition among health care professionals who view harm reduction as counter to 'curative' approaches to care (Pauly, 2008). In addition, drug legislation may preclude the adoption of comprehensive harm reduction approaches in some jurisdictions (Beletsky et al., 2008). It may, therefore, be instructive to position harm reduction as an 'ethical approach' intended to minimize harm as part of a broader strategy to ensure equitable access to hospital care (Pauly, 2008), and thereby locate it within the scope of health care practice. This approach has previously been used by an HIV/AIDS care facility in a Canadian setting to reposition supervised injection services as an issue of ethical health care practice (Wood et al., 2003). Furthermore, involving PWID in the development and implementation of hospital-based harm reduction services may serve to increase the acceptability of such services while promoting agency among PWID, and thus an opportunity to work in a productive fashion with hospital staff.

This study has limitations that should be taken into consideration. Because our participants had been discharged against medical advice, their experiences in hospital may be negatively biased, and may not be representative of those who completed treatment. Our findings are 
also specific to hospitals in the Vancouver area and, although they generate insights that may be relevant to other settings where hospital care is shaped by similar contextual forces, they cannot fully account for PWID's experiences in hospitals. Whereas our participants were covered by universal, publicly-funded health care insurance, PWID in other settings may face additional financial barriers to care that have an additional impact on hospital care. Finally, because we did not interview hospital staff, our findings represent only the perspectives of PWID. It is, therefore, possible that we overlooked some of the contextual forces that shaped specific aspects of care. Further research into the perspectives of hospital staff regarding the care of injection drug-using populations, and the potential integration of harm reduction services into hospitals, is urgently needed.

In conclusion, this study documents how hospitals constitute a ‘risk environment' for PWID. Our findings demonstrate that contextual forces operating within hospital settings foster conditions that increase the potential for drug and health harms, including discharges against medical advice. Optimizing evidence-based drug and pain treatment services, augmented by comprehensive harm reduction services, have significant potential to promote health equity by reshaping the environmental context of hospital care, and thereby reducing the enormous health and fiscal impacts resulting from discharges against medical advice.

\section{Acknowledgments}

The authors thank the study participants for their contribution to the research, as well as current and past researchers and staff with the British Columbia Centre for Excellence in HIV/AIDS. Tricia Collingham, Cameron Dilworth, Ivan Fletcher, Jennifer Matthews and Aaron McKinney provided research support and assistance. This study was supported by the Canadian Institutes of Health Research (MOP-81171) and the US National Institutes of Health (R01DA033147). This research was undertaken, in part, thanks to funding from the Canada Research Chairs program through a Tier 1 Canada Research Chair in Inner City Medicine which supports Dr. Evan Wood. Drs. Thomas Kerr, Ryan McNeil and Will Small are supported by the Michael Smith Foundation for Health Research.

\section{References}

Aceijas C, Rhodes T. Global estimates of prevalence of HCV infection among injecting drug users. International Journal of Drug Policy. 2007; 18(5):352-358. [PubMed: 17854722]

Anis AH, Sun H, Guh DP, Palepu A, Schechter MT, O'Shaughnessy MV. Leaving hospital against medical advice among HIV-positive patients. Canadian Medical Association Journal. 2002; 167(6): 633-637. [PubMed: 12358196]

Beletsky L, Davis CS, Anderson E, Burris S. The law (and politics) of safe injection facilities in the United States. American Journal of Public Health. 2008; 98(2):231-237. [PubMed: 18172151]

Berg KM, Arnsten JH, Sacajiu G, Karasz A. Providers' experiences treating chronic pain among opioid-dependent drug users. Journal of General Internal Medicine. 2009; 24(4):482-488. [PubMed: 19189194]

British Columbia College of Physicians and Surgeons. Prescription Review Committee Prescribing Principles. Author; Vancouver, BC: 2012.

Chan AC, Palepu A, Guh DP, Sun H, Schechter MT, O'Shaughnessy MV, et al. HIV-positive injection drug users who leave the hospital against medical advice: The mitigating role of methadone and social support. Journal of Acquired Immune Deficiency Syndromes. 2004; 35(1):56-59. [PubMed: 14707793]

Choi M, Kim H, Qian H, Palepu A. Readmission rates of patients discharged against medical advice: A matched cohort study. PLoS ONE. 2011; 6(9):e24459. [PubMed: 21931723]

Cooper H, Brady JE, Ciccarone D, Tempalski B, Gostnell K, Friedman SR. Nationwide increase in the number of hospitalizations for illicit injection drug use-related infective endocarditis. Clinical Infectious Diseases. 2007; 45(9):1200-1203. [PubMed: 17918083]

Corbin, JM.; Strauss \& Strauss, AL. Basics of qualitative research: Techniques and procedures for developing grounded theory. 3rd ed.. Sage Publications; Los Angeles, Calif: 2008. 
Fairbairn N, Milloy MJ, Zhang R, Lai C, Grafstein E, Kerr T, et al. Emergency department utilization among a cohort of HIV-positive injecting drug users in a Canadian setting. The Journal of Emergency Medicine. 2011; 43(2):236-243. [PubMed: 21719229]

Fairbairn N, Small W, Shannon K, Wood E, Kerr T. Seeking refuge from violence in street-based drug scenes: Women's experiences in North America's first supervised injection facility. Social Science \& Medicine. 2008; 67(5):817-823. [PubMed: 18562065]

Gebo KA, Diener-West M, Moore RD. Hospitalization rates differ by hepatitis C status in an urban HIV cohort. Journal of Acquired Immune Deficiency Syndromes. 2003; 34(2):165-173. [PubMed: 14526205]

Glasgow JM, Vaughn-Sarrazin M, Kaboli PJ. Leaving against medical advice (AMA): Risk of 30-day mortality and hospital readmission. Journal of General Internal Medicine. 2010; 25(9):926-929. [PubMed: 20425146]

Hwang SW, Li J, Gupta R, Chien V, Martin RE. What happens to patients who leave hospital against medical advice? Canadian Medical Association Journal. 2003; 168(4):417-420. [PubMed: 12591781]

Kerr T, Palepu A. Safe injection facilities in Canada: Is it time? Canadian Medical Association Journal. 2001; 165(4):436-437. [PubMed: 11531053]

Kerr T, Small W, Hyshka E, Maher L, Shannon K. 'It's more about the heroin': Injection drug users' response to an overdose warning campaign in a Canadian setting. Addiction. 2013; 108(7):12701276. [PubMed: 23551565]

Kerr T, Wood E, Grafstein E, Ishida T, Shannon K, Lai C, et al. High rates of primary care and emergency department use among injection drug users in Vancouver. Journal of Public Health. 2005; 27(1):62-66. [PubMed: 15564279]

Krusi A, Small W, Wood E, Kerr T. An integrated supervised injecting program within a care facility for HIV-positive individuals: A qualitative evaluation. AIDS Care. 2009; 21(5):638-644. [PubMed: 19444673]

Lloyd-Smith E, Wood E, Zhang R, Tyndall MW, Montaner JS, Kerr T. Risk factors for developing a cutaneous injection-related infection among injection drug users: A cohort study. BMC Public Health. 2008; 8:405. [PubMed: 19068133]

Lopez AM, Bourgois P, Wenger LD, Lorvick J, Martinez AN, Kral AH. Interdisciplinary mixed methods research with structurally vulnerable populations: Case studies of injection drug users in San Francisco. International Journal of Drug Policy. 2013; 24(2):101-109. [PubMed: 23312109]

Mathers BM, Degenhardt L, Phillips B, Wiessing L, Hickman M, Strathdee SA, et al. Global epidemiology of injecting drug use and HIV among people who inject drugs: A systematic review. The Lancet. 2008; 372(9651):1733-1745.

Mathers BM, Degenhardt L, Phillips B, Wiessing L, Hickman M, Strathdee SA, et al. Global epidemiology of injecting drug use and HIV among people who inject drugs: A systematic review. The Lancet. 2008; 372(9651):1733-1745.

McInnes CW, Druytz E, Harvard SS, Gilbert M, Tyndall MW, Lima VD, et al. HIV/AIDS in Vancouver, British Columbia: A growing epidemic. Harm Reduction Journal. 2009; 6(5)

Merrill JO, Rhodes LA, Deyo RA, Marlatt GA, Bradley KA. Mutual mistrust in the medical care of drug users: The keys to the "narc" cabinet. Journal of General Internal Medicine. 2002; 17(5):327333. [PubMed: 12047728]

Millar, J. HIV, hepatitis, and injection drug use in British Columbia - pay now or pay later?. British Columbia Ministry of Health; Victoria, BC: 1998.

Miller NS, Sheppard LM, Colenda CC, Magen JD. Why physicians are unprepared to treat patients who have alcoho- and Drug-related disorders. Academic Medicine. 2001; 76(5):410-418. [PubMed: 11346513]

Neale J, Tompkins C, Sheard L. Barriers to accessing generic health and social care services: A qualitative study of injecting drug users. Health \& Social Care in the Community. 2008; 16(2): 147-154. [PubMed: 18290980]

Neighbor ML, Dance TR, Hawk M, Kohn MA. Heightened pain perception in illicit substance-using patients in the ED: Implications for management. The American Journal of Emergency Medicine. 2011; 29(1):50-56. [PubMed: 20825774] 
Palepu A, Tyndall MW, Leon H, Muller J, O'Shaughnessy MV, Schechter MT, et al. Hospital utilization and costs in a cohort of injection drug users. Canadian Medical Association Journal. 2001; 165(4):415-420. [PubMed: 11531049]

Passik SD, Kirsh KL, Donaghy KB, Portenoy RK. Pain and aberrant drug-related behaviors in medically ill patients with and without histories of substance abuse. The Clinical Journal of Pain. 2006; 22(2):173-181. [PubMed: 16428952]

Pauly B. Shifting moral values to enhance access to health care: Harm reduction as a context for ethical nursing practice. International Journal on Drug Policy. 2008; 19(3):195-204. [PubMed: 18467086]

Providence Health Care. Patient rights + responsibilities: Safety during your stay. N.DRetrieved April 10, 2013, from http://www.providencehealthcare.org/hospitals-residences/st-pauls-hospital/infopatients-families/patient-rights-responsibilities/safety

QSR International. NVivo qualitative data analysis software. Version 8. 2008.

Quesada J, Hart LK, Bourgois P. Structural vulnerability and health: Latino migrant laborers in the United States. Medical Anthropology. 2011; 30(4):339-362. [PubMed: 21777121]

Rachlis BS, Kerr T, Montaner JS, Wood E. Harm reduction in hospitals: Is it time? Harm Reduction Journal. 2009; 6:19. [PubMed: 19638238]

Rhodes T. The 'risk environment': A framework for understanding and reducing drug-related harm. International Journal of Drug Policy. 2002; 13(2):85-94.

Rhodes T. Risk environments and drug harms: A social science for harm reduction approach. International Journal of Drug Policy. 2009; 20(3):193-201. [PubMed: 19147339]

Rhodes T, Kimber J, Small W, Fitzgerald J, Kerr T, Hickman M, et al. Public injecting and the need for "safer environment interventions" in the reduction of drug-related harm. Addiction. 2006; 101(10):1384-1393. [PubMed: 16968336]

Rhodes T, Singer M, Bourgois P, Friedman SR, Strathdee SA. The social structural production of HIV risk among injecting drug users. Social Science \& Medicine. 2005; 61(5):1026-1044. [PubMed: 15955404]

Riddell C, Riddell R. Welfare checks, drug consumption, and health. Journal of Human Resources. 2006; XLI(1):138-161.

Scheper-Hughes, N. Death without weeping: The violence of everyday life in Brazil. University of California Press; Berkeley: 1992.

Shannon K, Kerr T, Allinott S, Chettiar J, Shoveller J, Tyndall MW. Social and structural violence and power relations in mitigating HIV risk of drug-using women in survival sex work. Social Science \& Medicine. 2008; 66(4):911-921. [PubMed: 18155336]

Small W, Rhodes T, Wood E, Kerr T. Public injection settings in Vancouver: Physical environment, social context and risk. International Journal of Drug Policy. 2007; 18(1):27-36. [PubMed: 17689341]

Small W, Wood E, Lloyd-Smith E, Tyndall M, Kerr T. Accessing care for injection-related infections through a medically supervised injecting facility: A qualitative study. Drug and Alcohol Dependence. 2008; 98(1-2):159-162. [PubMed: 18650034]

Strathdee SA, Patrick DM, Currie SL, Cornelisse PGA, Rekart ML, Montaner JSG, et al. Needle exchange is not enough: Lessons from the Vancouver injecting drug use study. AIDS. 1997; 11(8):F59-F65. [PubMed: 9223727]

Vancouver Coastal Health. Clinical practice document: Alcohol and illegal substances. Vancouver Coastal Health; Vancouver, Canada: 2008.

Vlahov D, Wientge D, Moore J, Flynn C, Schuman P, Schoenbaum EE. Violence among women with or at risk for HIV infection. AIDS \& Behavior. 1998; 2(1):53-60.

Wagner KD, Davidson PJ, Pollini RA, Strathdee SA, Washburn R, Palinkas LA. Reconciling incongruous qualitative and quantitative findings in mixed methods research: Exemplars from research with drug using populations. International Journal on Drug Policy. 2012; 23(1):54-61. [PubMed: 21680168]

Warner-Smith M, Darke S, Day C. Morbidity associated with non-fatal heroin overdose. Addiction. 2002; 97(8):963-967. [PubMed: 12144598] 
Wood E, Montaner JS, Yip B, Tyndall MW, Schechter MT, O'Shaughnessy MV, et al. Adherence and plasma HIV RNA responses to highly active antiretroviral therapy among HIV-1 infected injection drug users. Canadian Medical Association Journal. 2003; 169(7):656-661. [PubMed: 14517122]

Wood RA, Zettel P, Stewart W. The Dr. Peter Centre: Harm reduction nursing. The Canadian Nurse. 2003; 99(5):20-24. [PubMed: 12778610]

Yong TY, Fok JS, Hakendorf P, Ben-Tovim D, Thompson CH, Li JY. Characteristics and outcomes of discharges against medical advice among hospitalized patients. Internal Medicine Journal. 2013; 43(7):798-802. [PubMed: 23461391] 


\section{Highlights}

1. Examines social-structural production of hospital discharge against medical advice among people who inject drugs (PWID).

2. Pain and withdrawal management practices experienced as form of everyday violence.

3. Social-structural conditions in hospitals produce discharge against medical advice.

4. Policy and programmatic reforms are urgently needed to reorient hospital care for PWID.

5. Points to a need for evidence-based pain management augmented by harm reduction approaches. 